MS17-02

\section{Crystallization of biomimetic calcite aggregates in hydrogel systems}

Lurdes Fernández-Díaz', Erika Griesshaber², Xiaofei Yin², Fitriana Nindiyasari ${ }^{3}$, Martina Greiner ${ }^{2}$, Florian Weitzel ${ }^{2}$, Andreas Ziegler ${ }^{4}$, Wolfgang Schmah ${ }^{2}$

1. Department of Mineralogy and Petrology, Complutense University of Madrid (UCM) \& IGEO (ICMM, CSIC), Madrid, Spain

2. Department für Geo- und Umweltwissenschaften, LudwigMaximilians-Universität, Munich, Germany

3. NRG, Le Petten, Netherlands (Holland, Europe

4. Central Facility for Electron Microscopy, University of Ulm, Ulm, Germany

\section{email: Ifdiaz@geo.ucm.es}

The formation of biological hard tissues takes place in hydrous gelatinous environments rich in polysaccharides, proteins and glycoproteins. These organic components are arranged in compliant three-dimensional matrices that direct the crystallization of the mineral component, within which they become occluded as porous membranes and networks of fibrils. The composite nature of biological hard tissues together to their distinct hierarchical architecture provide them with enhanced mechanical properties that are highly desirable in man-made materials. Designing successful routes to produce high-performance bio-inspired organic-inorganic composites relies on our understanding of the parameters that control the occlusion of organic matrices within growing crystals and the effect that this occlusion has on the micro-structuring of the mineral. Artificial hydrogels that have traditionally been used as crystallization platforms to produce large crystals of sparingly soluble phases share numerous features with the organic matrices found in hard tissues. Interestingly, these hydrogels also become occluded within crystals during growth. The way of gel occlusion is distinct and relates to characteristics that are specific of the type of hydrogel, like the nature of the interactions that hold its matrix together and its porosity. ${ }^{1}$ Gel occlusion is further modulated by physicochemical conditions in the system, which determine supersaturation and growth rate, and their evolution. ${ }^{2}$ Here an overview of the morphological and microstructural characteristics, as uncovered by Field Emission Gun Scanning Electron Microscopy (FEGSEM) and Electron Backscattered Diffraction (EBSD), of gel-calcite composites grown in a variety of gels (silica, gelatin, agar, agarose, and gelatin-agarose mixtures) is presented. Differences in amount and organization of occluded gel polymeric matrices within the composites are discussed taking into consideration the gel mechanical response to crystallization pressure. Finally, a correlation between the characteristics of occluded gel distribution and microstructural features of the composites is stablished.

References:

[1] F. Nindiyasari, A. Ziegler, E. Griesshaber, L. Fernández-Díaz, J. Huber, P. Walther P, W. W. Schmahl. Cryst. Growth Des. 2015, $15,2667$.

[2] M. Greiner, X. Yin, L. Fernández-Díaz, E. Griesshaber, F. Weitzel, A. Ziegler, S. Veintemillas-Verdaguer, W. W. Schmahl. Cryst. Growth Des. 2018, 18, 1401.

Keywords: Biomimetic crystallization, Calcite, EBSD
MS17-03

\section{Induced nucleation of biomimetic calcium phosphates on 3D-printed porous polymer micro-scaffolds}

Jaime Gómez-Morales ${ }^{1}$, Luis Antonio González-Ramírez' , Youen

Vitry $^{2}$, Pierre Lambert ${ }^{2}$, Isaac Rodríguez-Ruiz ${ }^{3}$

1. Laboratorio de Estudios Cristalográficos, Instituto Andaluz de Ciencias de la Tierra. CSIC-Universidad de Granada, Armilla, Spain

2. Transferts Interfaces et Procédés, Université Libre de Bruxelles, Brussels, Belgium

3. Research Department on Mining and Fuel Reprocessing Processes. CEA, DEN, Bagnols-sur-Cèze, France

email: jaime@lec.csic.es

Heterogeneous nucleation and growth of calcium phosphates (hereafter $\mathrm{CaP}$ ) on polymer substrates is of great relevance for the preparation of bioactive scaffolds for non-load bearing applications in bone tissue engineering. ${ }^{1}$ In the last years we have developed a new synthetic route to precipitate biomimetic apatite (Ap) nanoparticles, which has been employed to deposit $\mathrm{CaP}$ films on mica muscovite sheets. ${ }^{2}$ The precipitation process, based on the vapour diffusion sitting drop micro-method, is performed in a closed two-chamber environmental micro-reactor called crystallization mushroom. ${ }^{2}$ The deposition of $\mathrm{CaP}$ is here driven by diffusion of both $\mathrm{CO}_{2}$ and $\mathrm{NH}_{3}$ (generated in situ by decomposition of $\mathrm{NH}_{4} \mathrm{HCO}_{3}$ ) through microdroplets containing $\mathrm{Ca}^{2+}$ and $\mathrm{HPO}_{4}{ }^{2-}$ ions, where substrates are dipped.

In this research ${ }^{3}$, preliminary precipitation experiments have been carried out using as model supports flat lamellae of different commercial polymers such as OSTEMER, SU-8 and PDMS and the polymer-ceramic composites ORMOCORE ${ }^{\mathrm{TM}}$ and ORMOCLAD ${ }^{\mathrm{TM}}$, all of them allowing 2D and 3D microfabrication techniques. Optimal reagent concentrations of the $40 \mu \mathrm{L}$ aqueous droplets inside the mushroom were $50 \mathrm{mM}$ $\mathrm{Ca}\left(\mathrm{CH}_{3} \mathrm{COO}\right)_{2}+30 \mathrm{mM}\left(\mathrm{NH}_{4}\right)_{2} \mathrm{HPO}_{4}(\mathrm{Ca} / \mathrm{P}=5 / 3)$ whereas $3 \mathrm{~mL}$ of a $40 \mathrm{mM} \mathrm{NH}_{4} \mathrm{HCO}_{3}$ solution were put in the chamber acting as reservoir. Under these conditions, and after 7 days of vapour diffusion, the deposited $\mathrm{CaP}$ films presented a composition of apatite plus octacalcium phosphate (OCP) in some of them, and different thicknesses, as confirmed by SEM, XRD and Raman spectroscopy.

In a second step, several prototypes of 3D printed scaffolds of cubic shape and $\sim 1 \mathrm{~mm}$ long, have been CAD-designed and prepared by the direct laser writing (DLW) technique using an IP-L 780 photoresist. These scaffolds are endowed of uniaxial and interconnected porosity with a controlled pore size within the range $25 \mu \mathrm{m}-100 \mu \mathrm{m}$. The $\mathrm{CaP}$ nucleation experiments, using these specimens as supports, were carried out using the same biomimetic route under similar reagent concentrations as determined before, and time of about 30 days. Prior to mineralization the scaffolds were dipped in a $0.1 \mathrm{M} \mathrm{HNO}_{3}$, then they were rinsed with deionized water and the intraporous air removed by means of a vacuum pump. The results show the full mineralization of the scaffolds, both externally and internally inside the pores, coating the full available surface area. It is concluded that combining the 3D DLW technique with this biomimetic route is a promising methodology to prepare hybrid bioactive mineralized porous scaffolds for some valuable ad-hoc applications in bone tissue engineering. 
References:

[1] Amini, A. R., Laurencin, C. T. \& Nukavarapu, S. P. (2012). Crit Rev Biomed Eng. 40(5), 363-408..

[2] Gómez-Morales, J., Verdugo-Escamilla, C. \& Gavira, J. A.

(2016) Cryst. Growth \& Des. 16, 5150-5158.

[3] JGM, LAGR and IRR thank the project Biomin-Nanoapatite (MAT2014-60533-R from Spanish MINEICO and FEDER). B.H. and Y.V. thank PAI 7/38

MicroMAST et FNRS through the GEQ 22687275 project "3D

microstructuration and microengineering of surfaces with 2 photon lithography".

Keywords: calcium phosphates, polymer scaffolds, 3D-microprinting

\section{MS17-04}

\section{The directing effects of bacterial EPS and artificial hydrogel matrices on calcite crystal organization in EPS-hydrogel- calcite composite aggregates}

Xiaofei Yin ${ }^{1}$, Florian Weitzel ${ }^{1}$, Erika Griesshaber ${ }^{1}$, Concepción

Jiménez-López ${ }^{2}$, Lurdes Fernández-Díaz ${ }^{3}$, Andreas Ziegler ${ }^{4}$,

Alejandro Rodríguez-Navarro ${ }^{5}$, Wolfgang. W. Schmahl ${ }^{1}$

1. Department of Geo- and Environmental Sciences, LudwigMaximilians-Universität München, Munich, Germany

2. Department of Microbiology, Universidad de Granada, Granada, Spain

3. Department of Crystallography and Mineralogy, Universidad Complutense de Madrid, Madrid, Spain

4. Central Facility for Electron Microscopy, Universität Ulm, Ulm, Germany

5. Department of Mineralogy and Petrology, Universidad de Granada, Granada, Spain

email: xiaofei.yin@campus.Imu.de

Mineralized structures generated under biological control are hierarchical composites that consist of two distinct materials: a compliant biopolymer matrix that is reinforced by stiff and hard minerals. In gastropod, bivalve, brachiopod shells the biopolymer matrix consists of two components: (i.) matrix membranes that subdivide space and influence shape and size of mineral units, (ii.) foam-like networks of fibrils occluded within the mineral units that control attachment and orientation of crystallites. Microbial cells surround themselves with a gelatinous coating: EPS (extracellular polymeric substances) that consists of polysaccharides, proteins, lipids and occurs in a wide range of molecular sizes, conformations, and physical/chemical properties. The EPS fabric is a three-dimensional fibrous scaffold that protects bacterial cells and allows them to orient themselves. Numerous bacteria precipitate carbonate as a by-product of their metabolic activity. The latter induces supersaturation with respect to carbonate phases in microenvironments within the EPS fabric ${ }^{[1]}$.

Recent studies ${ }^{[2]}$ have shown that in biocarbonate hard tissues organic membranes and fibres control mineral organization within the basic mineral units. The assembly pattern of the biomineral is adjusted according to the fabric of the preformed organics. In order to understand the influence of biopolymer membranes and fibres on mineral organization, we conducted EPS/agar gel/calcite growth experiments and investigated membrane formation, mineral/EPS/agar gel interlinkage and mineral organization in the composite aggregates. We used EPSs secreted by four different bacteria: the gram-positive bacterium Bacillus subtilis and the gram-negative bacteria Pseudomonas putida, Mycobacterium phlei and Mycobacterium smegmatis. Characterization was performed with HR-SEM imaging; patterns of mineral orientation were measured with electron backscatter diffraction (EBSD).

The EPS scaffold exerts a significant influence on aggregate morphology, the presence of membranes and their distribution within the aggregate and calcite crystal organization in the composites (Fig. 1). Membrane formation is extensive and is highly increased relative to composite aggregates grown without EPS. Rhombohedral morphologies are ab- 